\section{Intravenous conscious sedation training for the whole team}

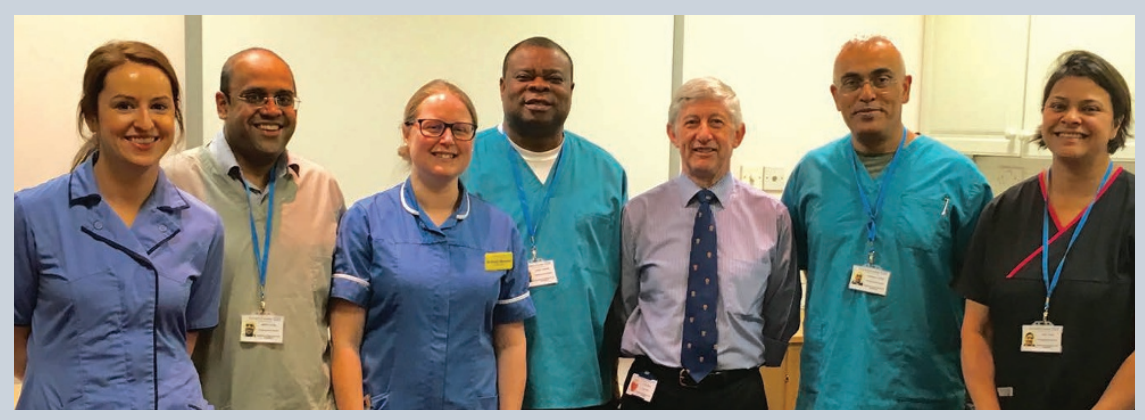

(I-r) Sheena Quille (dental nurse), Amish Patel (course member), Emily Sherwin (supervisor), Chris Vondee (course member), David Craig (supervisor), Parimal Patel (course member), and Lena Vakil (course member)

$\mathrm{I}$ n April 2015 the Intercollegiate Advisory Committee for Sedation in Dentistry (IACSD) of the Dental Faculties of the Royal Colleges of Surgeons and the Royal College of Anaesthetists published new Standards for conscious sedation in the provision of dental care which includes updated recommendations on conscious sedation training for the whole dental team. The Standards state that 'Patients have the right to expect a high quality service to meet their dental needs. This can only be achieved through robust, validated education and training of the entire dental team.'

As a result, an innovative course was established by Health Education England, London and the Department of Sedation \& Special Care Dentistry at Guy's and St Thomas' NHS Foundation Trust.

The new course was based on the learning outcomes outlined in the Standards and was run over eight days. The first two days were non-clinical and dedicated to increasing and updating theoretical knowledge and improving practical sedation skills. Topics covered included relevant physiology and pharmacology, patient assessment and treatment planning, cannulation, administration of midazolam, sedation monitoring and managing sedation related emergencies. The next six days were dedicated to treating patients requiring a wide range of dental treatment under intravenous sedation with midazolam. All the participants were encouraged to bring a dental nurse to observe the sedation technique, patient management and procedural skills. The course provided the opportunity to treat 20 patients with intravenous sedation under closely supervised conditions. In addition there were opportunities to experience the use of inhalation sedation and intranasal midazolam sedation and to observe the use of conscious sedation using a propofol infusion. Attendees gained confidence in treatment planning patients for sedation and also took part in a seminar on cognitive behavioural therapy.

The course was organised and supervised by Emily Sherwin, Specialist in Special Care Dentistry, and David Craig, Consultant in Special Care Dentistry. Having four attendees allowed for close supervision on the clinics with readily available support to assist with any challenging cases. The course involved continuous clinical assessment with both informal and formal feedback including workplace-based assessments and an endof-course review. There was also an MCQ examination to assess theoretical knowledge.

The course is designed for 'new starters' in dental sedation wishing to progress to independent (unsupervised) practice but it would also be helpful for experienced dentists who wish to expand their skills. Its clinical focus makes this course ideal for primary dental care dentists who are interested in adding conscious sedation to their skill set.

By Amish Patel and Emily Sherwin

1. Standards for Conscious Sedation in the Provision of Dental Care. Report of the Intercollegiate Advisory Committee for Sedation in Dentistry. The Dental Faculties of the Royal Colleges of Surgeons and the Royal College of Anaesthetists, 2015.

\section{Urgent notice for foundation dentists}

The British Dental Association (BDA) is reminding all new graduates who have recently been allocated to a dental foundation training post in England or Wales starting on 1 September this year that they must apply to be included in the dental performers list before that date. The same applies to inclusion in the HSCB dental list in Northern Ireland. The PCS and Area Teams/ Health Boards then have three months to finalise the inclusion of names in the list.

For those in Northern Ireland applying to be on the dental list, full completion of the HS48 form and attendance at the HSCB Dental Information session is a pre-requisite to a DS number being issued.

Young dentists and their trainers/educational supervisors should also check with the deaneries and PCS/ATs/HBs whether the process for inclusion is moving forward smoothly, so that any problems can be clarified in good time and the inclusion is confirmed by the end of November at the very latest.

If trainers/supervisors or foundation dentists are becoming aware of any problems with this process, please get in touch with the $\mathrm{BDA}$ as soon as possible.

\section{Faculty supports faster cancer referrals}

The Faculty of General Dental Practice (UK) has expressed its support for a new standards document on cancer referrals from the National Institute for Health and Care Excellence (NICE).

In the UK, over 20 people a day are diagnosed with oral cancer, and FGDP(UK) is asking dentists to take note in particular of the recommendation in the new document that 'people with suspected cancer who are referred to a cancer service are given written information encouraging them to attend'. The Faculty is recommending that dental practices make suitable resources, such as the NHS's 'Patient information for urgent referrals' leaflet, available for patients.

To download the leaflet visit: https://www. myhealth.london.nhs.uk/healthy-london/ cancer/pan-london-suspected-cancer-referrals/ patient-leaflets. 